\title{
Application of Multi-Attribute Crossplots to Tight Gas in X Area, Western China
}

\author{
Lifang Cheng, Yanchun Wang, Zhiguo Li, Fei Li, Fuxiu Gong \\ School of Geophysics and Information Technology, China University of Geosciences, Beijing, China \\ Email:chenglf@cugb.edu.cn
}

Received 17 March 2016; accepted 16 April 2016; published 19 April 2016

Copyright (C 2016 by authors and Scientific Research Publishing Inc.

This work is licensed under the Creative Commons Attribution International License (CC BY). http://creativecommons.org/licenses/by/4.0/

(c) (7) Open Access

\section{Abstract}

The gas-bearing reservoir in $\mathrm{X}$ area is mainly the tight sandstone reservoir characterized by low porosity and permeability, frequently lateral variation and poor connectivity of single sand. The previous research results reveal that the general seismic attributes analysis cannot meet the requirement of fluid identification. This is because the relationship between seismic attributes and their implication is uncertain and ambiguous, which decreases the precision of both reservoir prediction and fluid identification. To overcome the problem, multi-attribute crossplot technology is proposed from the mathematical statistical point of view rather than the correspondence between the seismic attributes and their geological implication. In this method, the wells which have the same statistical law are classified firstly, and then all the interest wells are retained while the wells beyond the statistical law are eliminated, and the seismic attributes sensitive to the same types of eliminated wells are optimized and used to generate crossplots. The nonzero area of their crossplots results just predicts the potential distribution. The discontinuity of subsurface geological conditions results in the non-continuous shape and the seismic bin lead to the mosaic form. The optimization of sensitive attributes relative to the same types of wells is independent from each other, and thus the order of attributes in crossplots does not affect the final prediction results. This method is based on the statistical theory and suitable for the areas such as the study area abundant of lots of well data. Application to $X$ area proves the effectiveness of this method and predicts plane distribution about different types of gas production. Due to the effect of faults and other geological factors, the partition prediction results using multi-attribute crossplots reach $\mathbf{9 5 \%}$ of coincidence which is obviously and far higher than the results of the whole area. The final prediction results show that the potential areas with medium and high gas production are mainly concentrated in the northern part of the study area, where lots of development research will be strengthened.

\section{Keywords}

Multi-Attribute Crossplots, Mathematical Statistics, Tight Sandstone Gas, Fluid Identification 


\section{Introduction}

The attribute crossplots analysis is commonly used to predict the reservoir and identify the fluid by analyzing the sensitive seismic attributes anomaly caused by reservoir for fluid via forward modeling. The crossplots indicates the approximate distribution of potential area. Different researchers of different times study either more seismic attributes sensitive to reservoir and fluid or advanced methods for computing crossplots to improve the reliability of attribute crossplots analysis in reservoir prediction and fluid identification. Reinaldo J. Michelena (2011) [1] proposed facies probabilities from multidimensional crossplots of seismic attributes and helped to improve sand identification where sands were more anisotropic than the background in the application of tight gas. Chao Wang (2015) [2] proposed two new seismic attribute technologies including anisotropy coherence technique (ACT) and fracture intensity inversion (FII) to provide an effective way to predict the distribution of DPF in similar geological settings. However, the attribute ambiguity reduces the accuracy of the predicted results. In this paper, we classify the wells having the same statistical laws from the mathematical statistical point of view rather than the corresponding relations between the seismic attributes and their geological implication, and predict the interest fluid according to gas-bearing properties of known wells included in the same statistical class.

\section{Method Theory}

Seismic attributes are the comprehensive response to the subsurface seismic and geological conditions. They have an uncertain relationship with their geological implication [3] [4]. The change of variable geological factors including lithology, geophysical properties, thickness, formation pressure and fluid properties, etc. may cause the anomaly of same seismic attribute, while the same attribute anomaly can be due to different geological factors. In addition, one single geological parameter can lead to anomalies of variable seismic attributes. It is the multi-to multi mapping relation that brings great difficulties to analyze the attributes anomaly only caused by different gas-bearing properties [5] [6].

Figure 1 shows a geological model with a sandstone layer of $30 \mathrm{~m}$ thickness, whose width and height is respectively $200 \mathrm{~m} 500 \mathrm{~m}$. The P-wave velocity and density of background mudstone are $3900 \mathrm{~m} / \mathrm{s}$ and 2340 $\mathrm{kg} / \mathrm{m}^{3}$, while these two parameters of sandstone are $4400 \mathrm{~m} / \mathrm{s}$ and $2530 \mathrm{~kg} / \mathrm{m}^{3}$. The seismic observation system is defined as the intermediate shot point where it is $100 \mathrm{~m}$ away from the left side of the model and bilateral receiver points whose distance between any two adjacent receivers is $5 \mathrm{~m}$. The shot point has a zero offset. The exploded wavelet is zero-phase Ricker wavelet with its main frequency at $40 \mathrm{~Hz}$. Keeping the top of sandstone layer unmoved, thickness non-isometrically varies from $5 \mathrm{~m}, 10 \mathrm{~m}, 15 \mathrm{~m}, 20 \mathrm{~m}$ to $30 \mathrm{~m}$. The corresponding synthetic seismic records of shot point after muting the first arrivals are shown in Figure 2(a), and the statistical amplitude spectrum of each trace in Figure 2(a) are shown in Figure 2(b). The main frequency decreases along with the thickness of sandstone increasing, and both bandwidth and attenuation gradient increase along with thickness increasing. Moreover, the amplitude of main frequency performs slight difference.

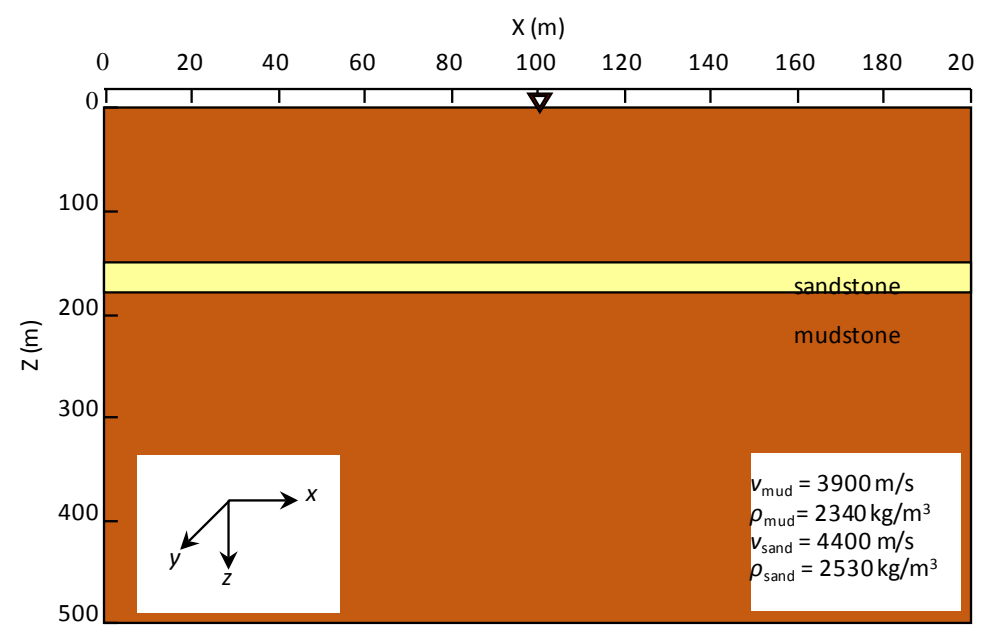

Figure 1. Geological sandstone model. The horizontal direction is the length of the model and the vertical one is the depth of the model. 


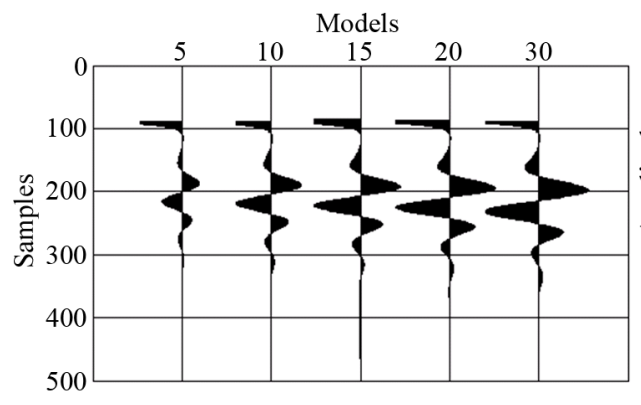

(a)

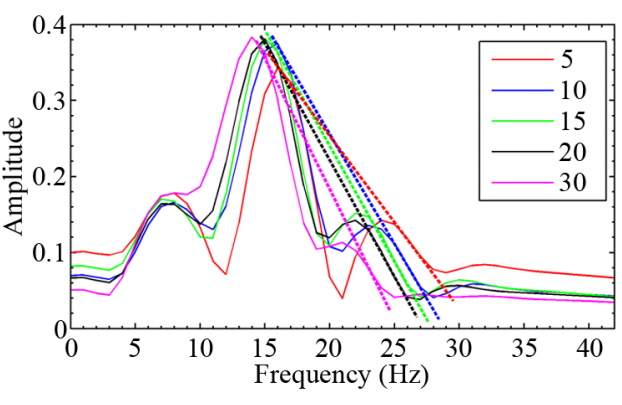

(b)

Figure 2. Synthetic CMP gathers after muting the first arrivals (a) and its statistical amplitude spectrum (b) of the central trace of different models. In figure (a), the x-axis represents the thickness of sandstone layer in different models and the y-axis shows the sampling number. In Figure (b), $\mathrm{x}$-axis is frequency in $\mathrm{Hz}$ and the $\mathrm{y}$-axis is normalized spectrum amplitude. The dashed lines resresent the attenuation gradient of different models.

Above forward modeling study shows that just different thickness can lead to the changes of these attributes including main frequency, main frequency amplitude, bandwidth and attenuation gradient, etc. Therefore, we propose the statistical analysis on the large number of sample data based on the mathematical statistical theory and optimize lots of attributes sensitive to different type of wells. This ultimate way is on the basis of that the wells with same production features have same statistical laws. Under the guidance of the idea that is retention and elimination, the crossplots of seismic attributes sensitive to the same type of wells provide the distribution of tight gas even high production gas. This method does not require mathematical theory and artificial geological implication of seismic attributes. The idea of retention and elimination means that in the process of screening the range of one sensitive attribute, the interest wells are fully retained, while the non-interest wells are eliminated as much as possible. Each sensitive attribute contributes to eliminating one or more non-interest wells. Crossplots of variable sensitive attributes help to retain the entire interest wells and eliminate all the non-interest ones. Multi-attribute crossplots avoid the ambiguity caused by single attribute, and get unique abnormal distribution of interest tight gas, and identify different gas-bearing characteristics according to the variable classification of interest wells.

\section{Application Examples}

\subsection{Introduction of Study Area}

$\mathrm{X}$ area is located in the east of Taibei depression in Turpan-Hami Basin, western China (in Figure 3(a)). The structural type is faulted anticline and the main gas-bearing reservoir is the tight sandstone intervals in the Sanjianfang group, Jurassic system. The main lithology is dark grey mudstone interbedded with thin fine sandstone as wells as siltstone and argillaceous siltstone (in Figure 3(b)) [7]-[9]. The reciprocal superposition between the braided delta sand body and faulted anticline structure formed lithologic reservoir developed on the tectonic setting. The reservoir in this area is characterized by the geophysical properties of low porosity, low permeability and low oil saturation, and by the geological properties of frequently lateral variation, development of isolated sand bodies and poor connectivity of single layer [10]. The distribution of oil and gas is affected by the structure, lithology and geophysical properties and so on. Previous research shows that gas-bearing properties of tight sandstone reservoir in $\mathrm{X}$ area are related to the abnormal morphology of seismic reflection. The downward pulling of seismic events probably indicates fluid, but not all anomalies reflect fluid even not all fluid are tight gas. Because the geological setting and seismic processing accuracy of different area differs each other, it cannot meet the requirements of fluid identification just using common attribute analysis. What gratified is that years of exploration and development have accumulated lots of well data in this area, and it provides convenient basis for fluid identification.

\subsection{Feasibility Analysis}

There are 44 wells in $\mathrm{X}$ area, production of all wells in the interest reservoir is classified to three types according 


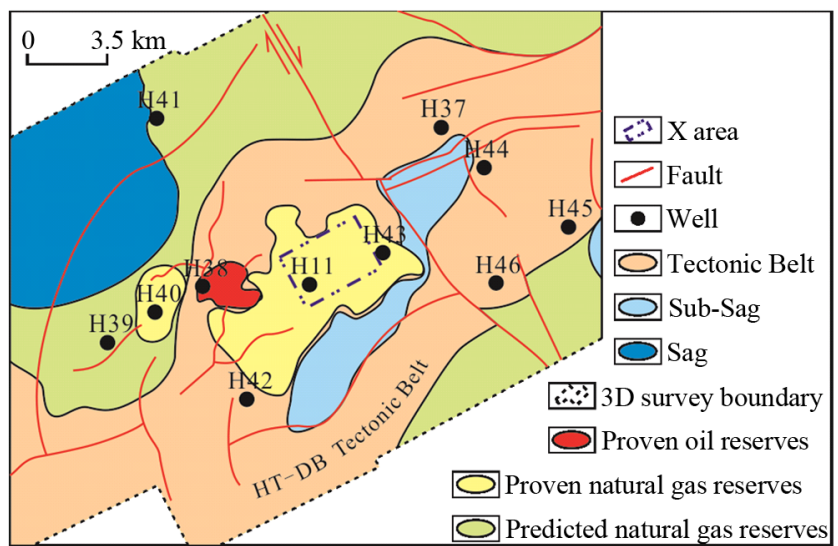

(a)

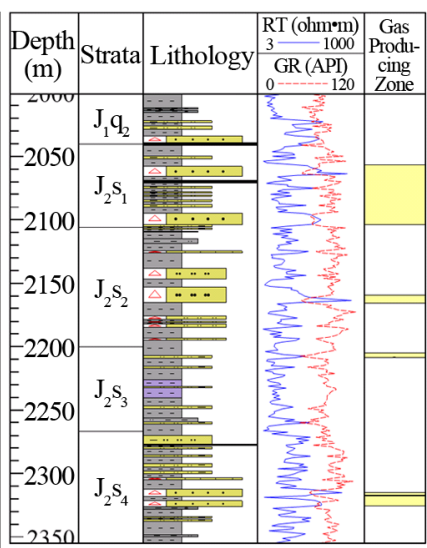

(b)

Figure 3. The Geographical strcuture location map (a) and lothological column (b) of X area. Figure 3ais referenced by Liu Juntian (2014).

to Table 1 . The classification results show that 17 wells are dry wells, and 21 wells are low production gas wells, and the rest 6 wells are medium and high production gas wells. Those wells are divided into sample wells and verification wells. There are two principles for selecting sample wells. One is that different production wells take up approximately similar proportion of every respective class, and the other is that sample wells are dispersed in every fault block. 24 wells are filtered for samples according to above principles and the reset 20 wells are used to verify the feasibility of this method.

Figure 4 shows some seismic attributes related to frequency of the interest reservoir. Those attributes are attenuation gradient, composite attenuation gradient, instantaneous bandwidth and instantaneous frequency respectively. Obviously any single attribute cannot completely distinguish various fluids and different types of production. Values at wells for each attribute are extracted, then the statistical results are shown in Figure 5. In Figure 5(a), the values of instantaneous bandwidth (IBW) at X19, X22 and X29 wells are lower than the values at all interest wells, so three wells are eliminated under the condition of retaining all interest wells using IBW attribute. Likewise, thin bed indicator (TBI) in Figure 5(b) as well as attenuation gradient (AG) in Figure 5(c) and composite attenuation gradient (CAG) in Figure 5(d) eliminate both X7 and X37 as well as X43 and X44 wells respectively. Four sensitive attributes including IBW, TBI and AG are selected after four turns of screening. Continuing comparison and analysis, seven sensitive attributes are finally optimized. Crossplots of these seven sensitive attributes indicate the gas-bearing distribution of interest reservoir in $\mathrm{X}$ area, which is shown in Figure 6(a). Crossplots of attributes sensitive to the medium and high production further predict the potential region of interest production shown in Figure 6(b). Using 20 verifying wells to verify the prediction results of medium and high production, the coincidence rate is $65 \%$ with three dry wells and three low production gas wells regarded as interest production and one medium production gas well eliminated.

\subsection{Partition Contrast}

The top structures of $\mathrm{J}_{2} \mathrm{~S}_{2}$ member shown in Figure 7 reflect that the entire area are divided into three parts by F1 and F5 faults, which are western part, central part and eastern part respectively. Most wells in the central area are medium and high production gas wells while the wells in the western and eastern areas are mainly low production gas and dry wells respectively. It may be fault sealing to fluid and sediment provenance that lead to the variance of gas production in different area. So it needs distributed prediction of gas-bearing anomalies. Figure 8 shows the partition identification results of medium and high production. After verification, the overall coincidence rate reaches up to $95 \%$, which is far more than $65 \%$ of the entire area. Above analysis prove that partition prediction has a better results and a higher accuracy.

Values of prediction map in Figure 8 display two numerical forms including zero values reflecting the bank portion and nonzero values reflecting the colorful area. The former explains that tight gas of those regions are rarely abundant in the interest reservoir, and the latter indicates the gas-bearing distribution with medium and high production. In addition, the map shows the form of discontinuity and mosaic. The discontinuity is caused 


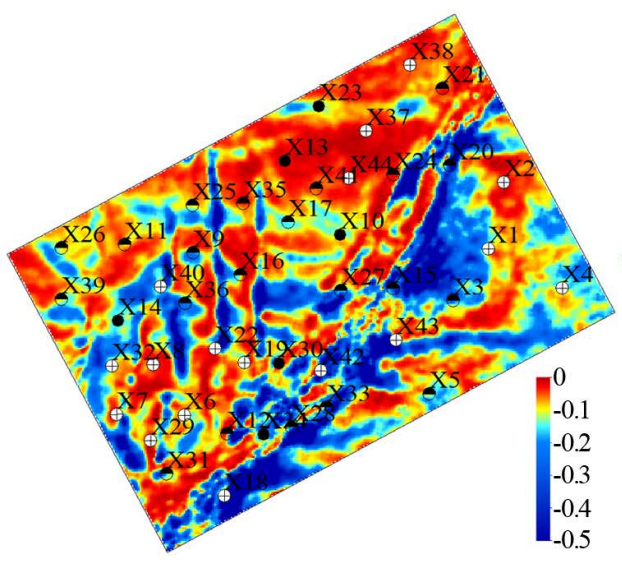

(a)

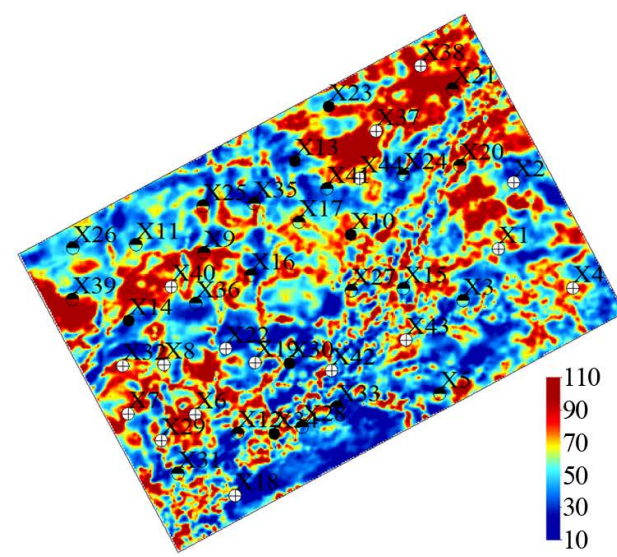

(c)

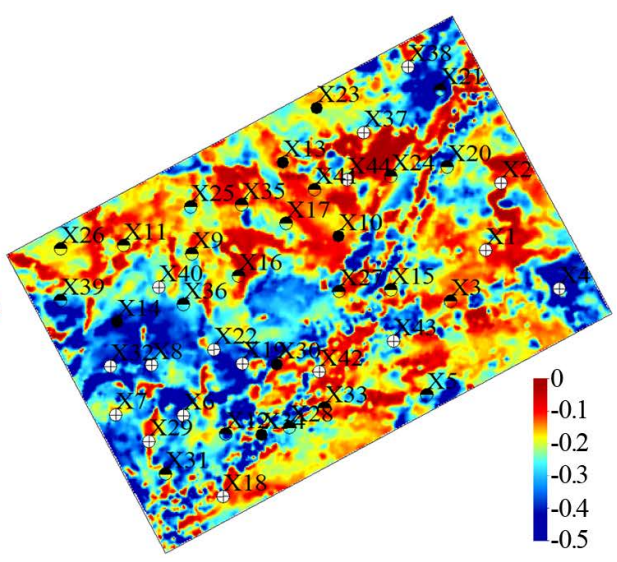

(b)

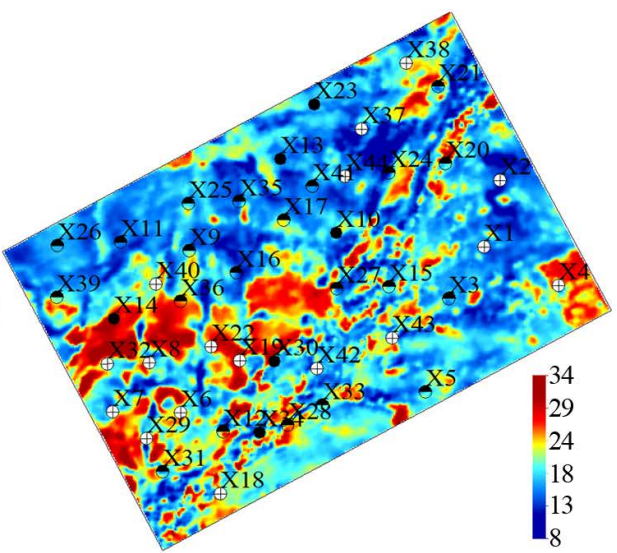

(d)

Figure 4. Some Seismic attributes of $\mathrm{J}_{2} \mathrm{~S}_{2}$ member in $\mathrm{X}$ area. (a) is attenuation gradient attribute, and (b) is composite attenuation gradient attribute, and (c) is instantaneous bandwidth attribute at $\mathrm{Hz}$, and (d) is instantaneous frequency attribute at $\mathrm{Hz}$.

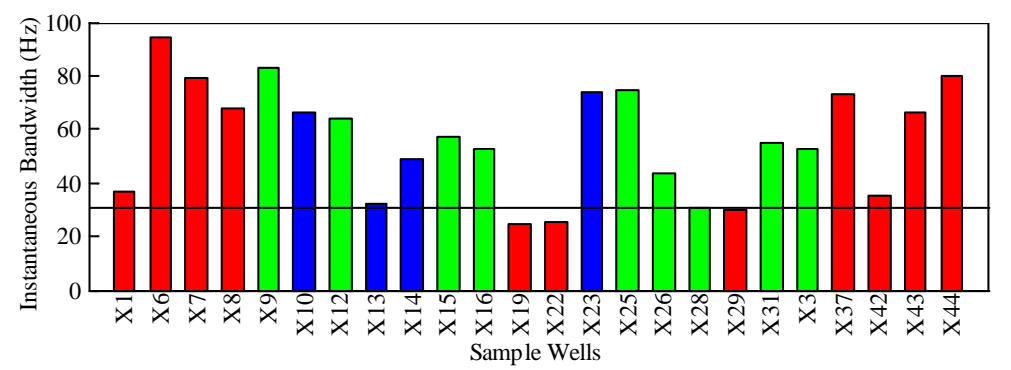

(a)

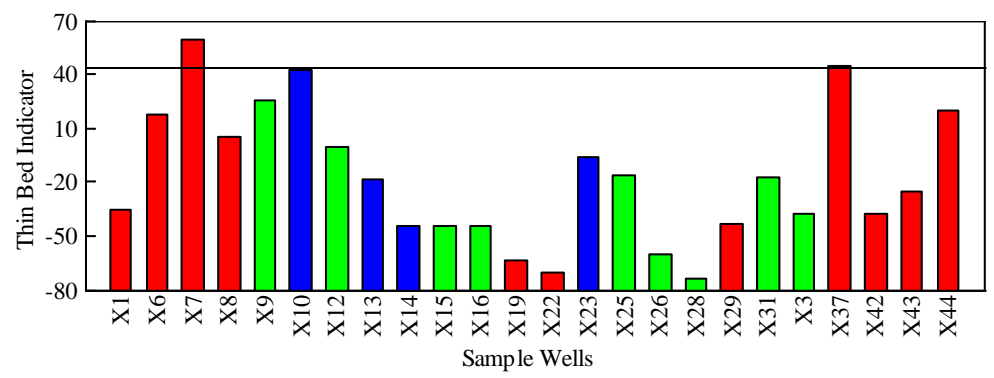

(b) 


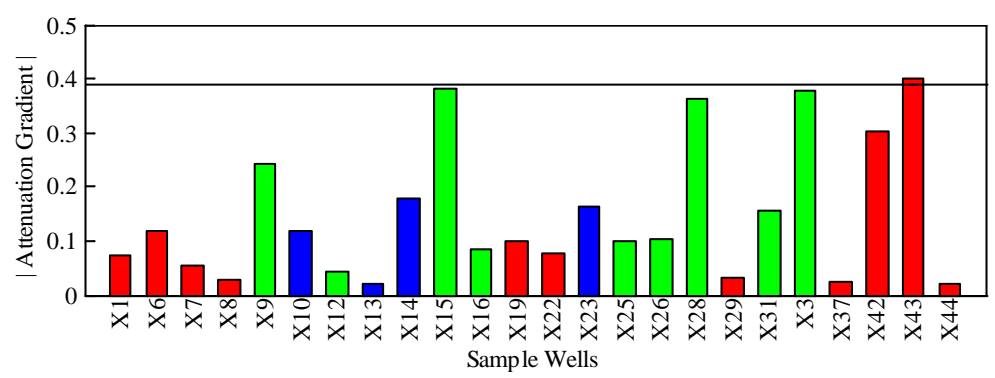

(c)

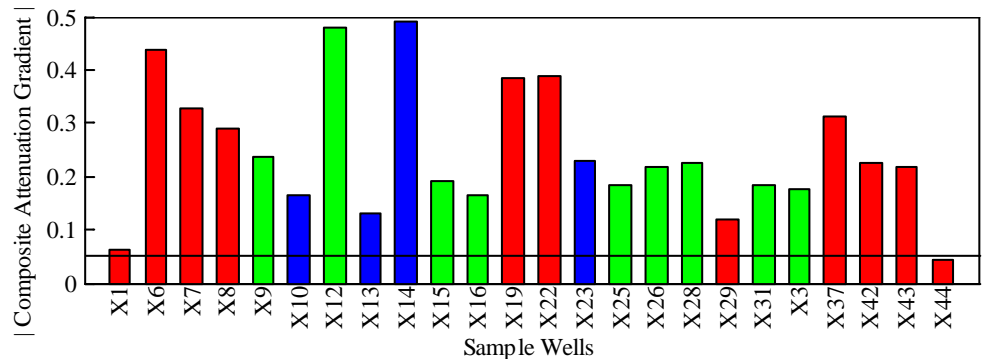

(d)

Figure 5. Optimization of seismic attributes sensitive to the gas-bearing wells using 24 sample wells. (a) is instantaneous bandwidth attribute, (b) is thin bed indicator, (c) is attenuation gradient attribute and (d) is .compositegradient attribute. Red histogram colums represent dry wells, and green bars are low production wells, and blue ones are used to represent the gas-bearing wells with medium and high production.

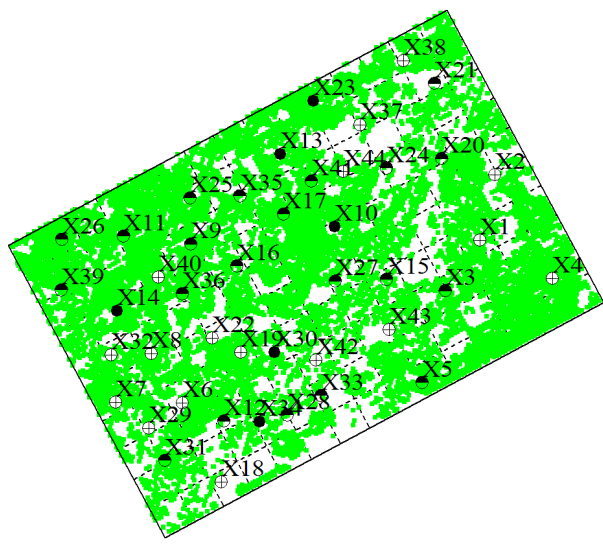

(a)

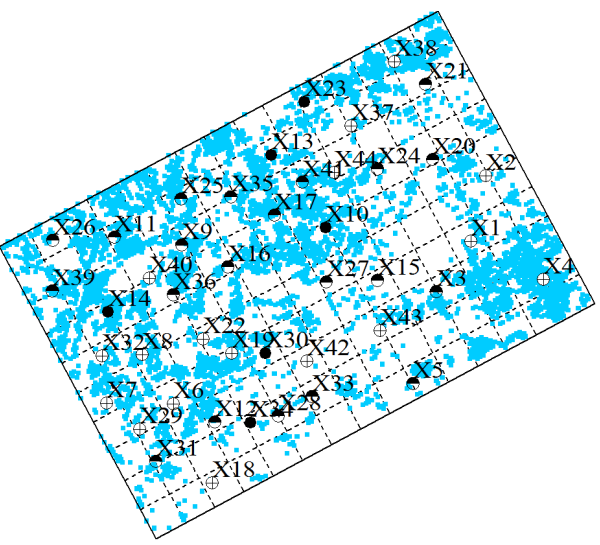

(b)

Figure 6. Map of gas-bearing anomaly (a) and medium and high production gas distribution (b) of $\mathrm{J}_{2} \mathrm{~S}_{2}$ member in X area.

Table 1. Standard of production classification in X area.

\begin{tabular}{cccc}
\hline class & low production & medium production & high production \\
\hline daily gas $\left(10^{4} \mathrm{~m}^{3}\right)$ & $<1$ & $1-2$ & $>2$ \\
\hline
\end{tabular}

by actual discontinuous geological conditions, even tight sandstone reservoir with frequent lateral variation and lots of isolated sand bodies, both of which make the dispersed form more obvious. The multi-attribute crossplots are used to identify fluid on the basis of seismic lines and traces. When a seismic bin of 3d data constituted by one line and one trace are discontinuous, the prediction results appears to mosaic. Because every sensitive attribute is independent in the process of selecting, crossplots of various attributes sensitive to the same kind of 


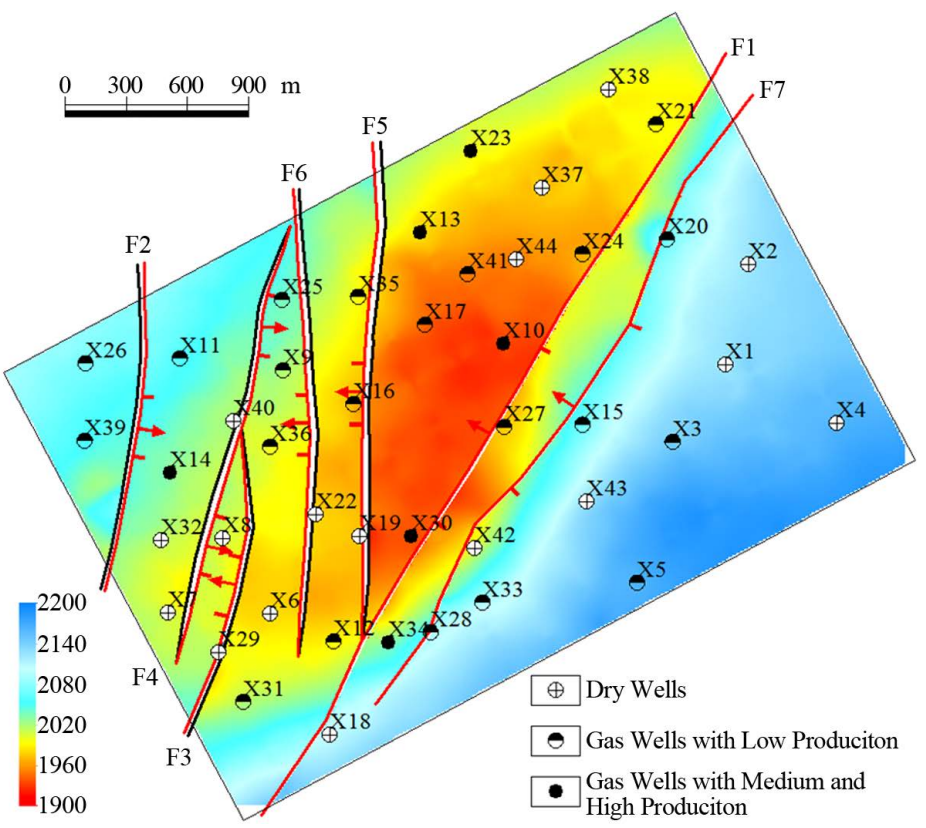

Figure 7. Structural interpretation result of the top of $\mathrm{J}_{2} \mathrm{~S}_{2}$ member in $\mathrm{X}$ area. Both F1 and F5 faults divide the entire area into three regions.

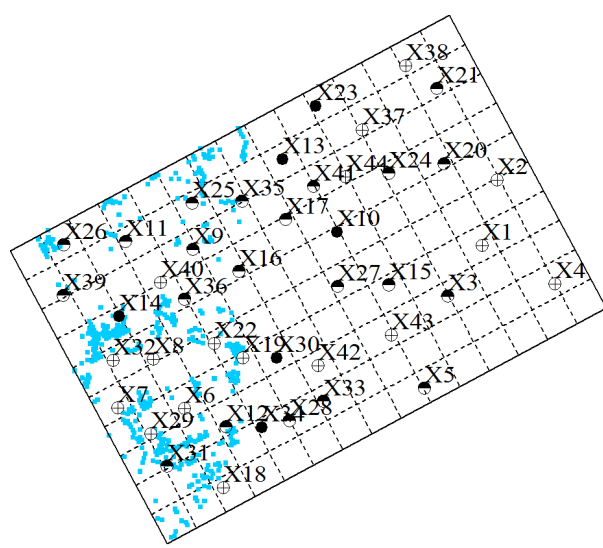

(a)

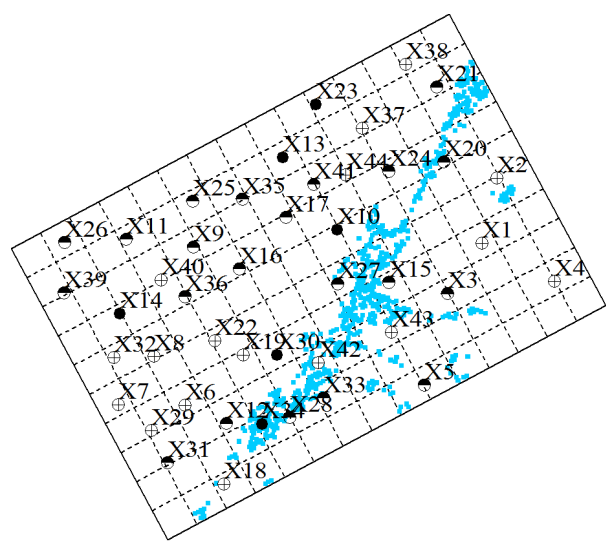

(c)

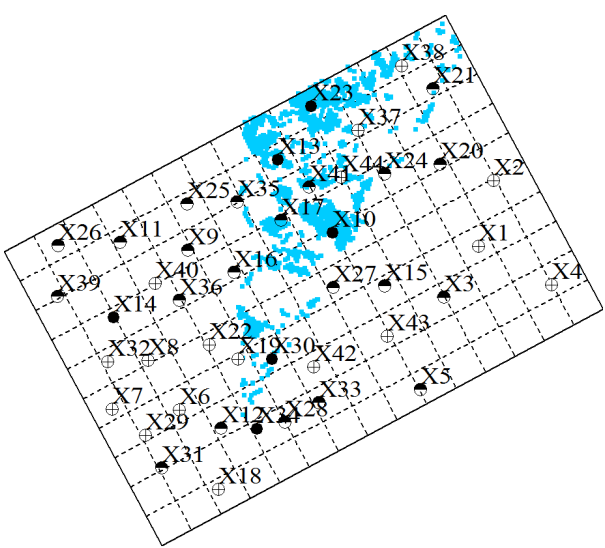

(b)

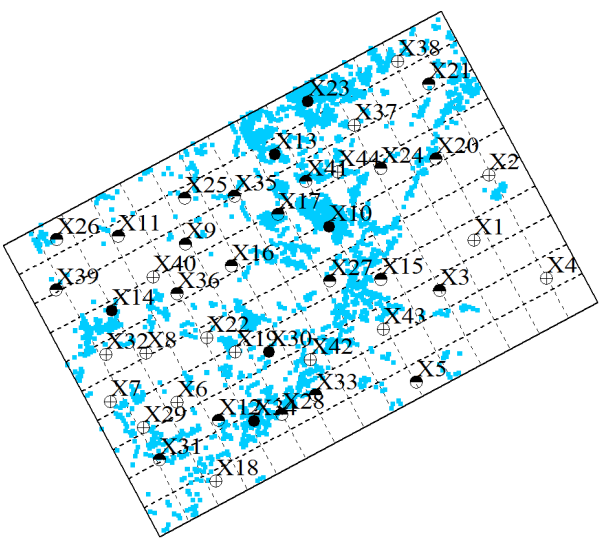

(d)

Figure 8. Distribution of medium and high production gas in the western (a), central (b), eastern (c) and the finally merged (d) area. 


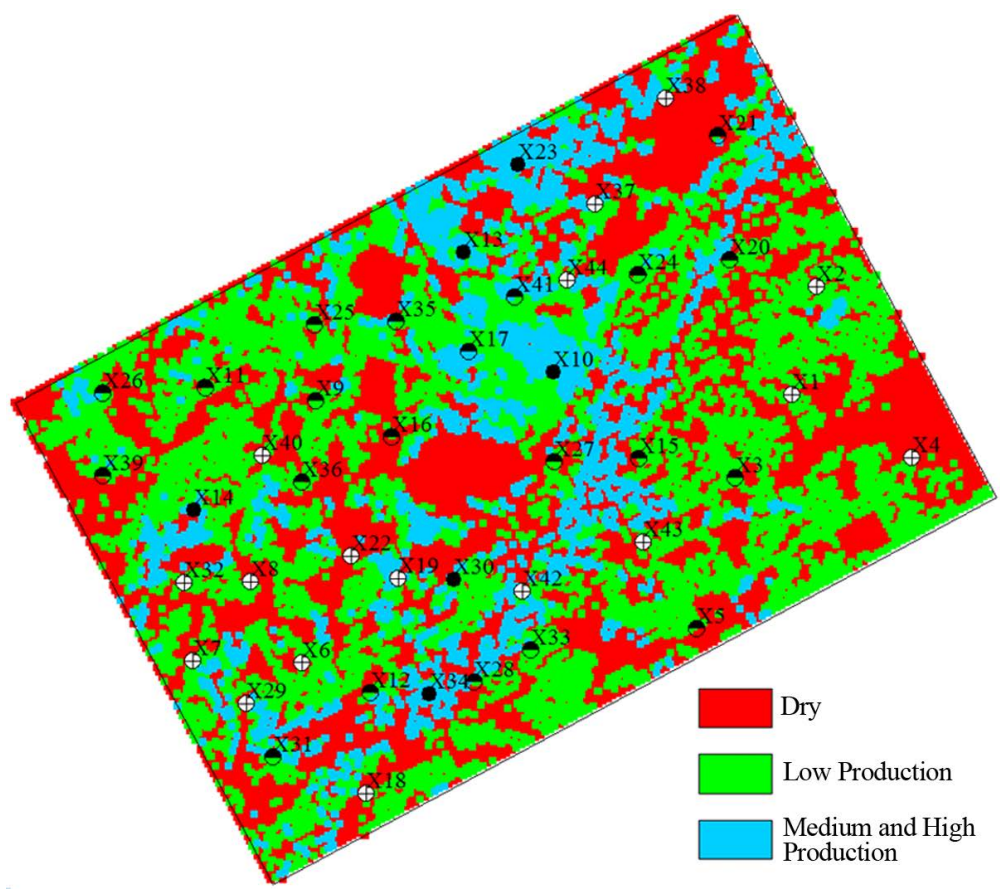

Figure 9. Anomaly distribution of tight gas in $\mathrm{X}$ area.

wells are regardless of orders.

\subsection{Prediction of Gas-Bearing Anomalies}

All wells of $X$ area are used as sample wells to optimize the sensitive attributes. The multi-attribute crossplots provide the distribution of gas-bearing anomalies with different production. Merged prediction results are shown in Figure 9. Different colors represent different anomalies. Regions with red color indicate dry reservoir, green regions describe the distribution of low production reservoir, and light blue ones are the potential gas-bearing areas with medium and high production. The potential areas are mainly located in the north of $\mathrm{X}$ area, and are the key goal for development.

\section{Conclusions}

1) Seismic attributes of reservoir don't correspond with their geological implication. Multi-attribute crossplots based on the mathematical statistics overcome the ambiguity caused by above problems. This method classifies the distribution having same statistical laws as a class and considers them having the same production properties.

2) The promoted method is suitable for areas with large numbers of well data. More sample wells can bring statistical regularity much more closing actual situation and more reliable predict outcomes.

3) The prediction results of this method contain zero values and nonzero values indicating the development of interest fluid. Frequent lateral variation of subsurface geological settings leads to the discontinuity of the distribution map. Non-continuous distribution of seismic bins which is the smallest unit of crossplots results in the mosaic forms. Moreover, the orders of crossplots of seismic attributes are sensitive to the same class of wells.

4) Combined with the actual structural situations of $X$ area, partition prediction improves the accuracy greatly compared with entire partition. Different areas do comparative analysis and adjustment according to their own situation.

\section{Acknowledgements}

The authors are very grateful to both of China University of Geosciences and Beijing Tech Art, Open Source S\&T Company that provided the needed resources, which made this research successful. Many thanks to the 
sponsor from Key Geophysical Technology Research Program on the Unconventional Oil and Gas of China National Petroleum Corporation (2014A-3612). Also, much appreciation is extended to the editor and the anonymous reviewers highly acknowledged for their critical comments and suggestions which helped to improve the quality of the original manuscript.

\section{References}

[1] Michelena, R.J., Godbey, K.S.G. and Rodrigues, P.E. (2011) Facies Probabilities from Multidimensional Crossplots of Seismic Attributes: Application to Tight Gas Mann Creek Field, Piceance Basin, Colorado. The Leading Edge, 30, 6269. http://dx.doi.org/10.1190/1.3535434

[2] Wang, C., Lu, Y.C. and Huang, H.G. (2015) New Seismic Attribute Technology for Predicting Dissolved Pore-Fracture of Deeply Buried Platform Margin Reef-Beach System in Northeast Sichuan Basin. Journal of Earth Science, 26, 373-383. http://dx.doi.org/10.1007/s12583-015-0540-0

[3] Gao, W.B. (2012) Applied Research of Reservoir Prediction in the Area of Hongtai. Master Thesis, Yangtze University, Wuhan.

[4] Alridha, N.A. and Muhsin, S.U. (2015) 3-D Seismic Attributes Analysis in Balad Oil Field-Center of Iraq. Arab Journal of Geosciences, 8, 2785-2798. http://dx.doi.org/10.1007/s12517-014-1389-8

[5] Miller, P., Dasgupta, S. and Shelander, D. (2012) Seismic Imaging of Migration Pathways by Advanced Attribute Analysis, Alaminos Canyon 21, Gulf of Mexico. Marine and Petroleum Geology, 34, 111-118. http://dx.doi.org/10.1016/j.marpetgeo.2011.09.005

[6] Chopra, S. and Marfurt, K.J. (2009) Detecting Stratigraphic Features via Crosslotting of Seismic Discontinuity Attributes and Their Volume Visualization. The Leading Edge, 28, 1422-1426. http://dx.doi.org/10.1190/1.3272695

[7] Chen, X.G., Xie, R.J. and Zhang, X. (2012) Fine Strata Classification and Correlation for Sanjianfang Formation (2s) in Hongtai Oil and Gas Field. Tuha Oil and Gas, 17, 25-27. (In Chinese)

[8] Li, W. (2011) The Research of Geologic Modeling of Gas Reservoirs in Hongtai 2 Block. Sino-global Energy, 16, 5153. (In Chinese)

[9] Bi, J.F., Liu, J.H. and Yang, Y. (2010) Depositional System of Braided River Delta in Hongtai Area of Tuha Basin. Fault-block Oil and Gas Field, 17, 702-705. (In Chinese)

[10] Ma, C., Xie, R.J. and Cai, Z.T. (2012) 3D Geological Modeling of the Gas Reservoir in Sanjianfang Formation of Hongtai Gas Field. Computer Applications of Petroleum, 49-51. (In Chinese) 